\title{
Radiographic Evaluation of Caudal Vena Cava Size as a Useful Parameter for the Diagnosis of Heart Disease in Dairy Cattle
}

\author{
JILINTAI ${ }^{1)}$, Sayaka HASHIYAMA ${ }^{2)}$, Yuhsuke GONDA ${ }^{1)}$, Hiroshi ISHIKAWA ${ }^{1)}$, Motoyoshi SATO $^{2)}$ and \\ Kazuro MIYAHARA ${ }^{1) *}$ \\ ${ }^{1)}$ Veterinary Medical Teaching Hospital and ${ }^{2)}$ Department of Clinical Veterinary Science, Obihiro University of Agriculture and \\ Veterinary Medicine, Obihiro, Hokkaido 080-8555, Japan
}

(Received 15 February 2006/Accepted 1 May 2006)

ABSTRACT. To quantify the radiographic parameters of the caudal vena cava (CVC) in healthy cattle and demonstrate their clinical usefulness, the present study compared the ratios of the diameter of the thoracic CVC to the diameter of the aorta (Ao) and length of the thoracic vertebrae (VL), which are all positioned in the same intercostal space, in 81 healthy control cattle (43 growing, 38 adult) and 10 cattle with heart disease. The average diameter of the CVC (CVCave) was correlated with the size of the Ao and VL in the control cows. Although the diameter and pulsation index of the CVC differed significantly between the growing and adult cows, the ratios of $\mathrm{CVC} / \mathrm{Ao}$ and $\mathrm{CVC} / \mathrm{VL}$ were fixed values for both the growing and mature cattle. However, in the cattle with heart disease, the pulsation index of the CVC was significantly lower or there was absence of pulsation due to a dilated CVC, and the ratio of CVCave/Ao and CVCave/VL were significantly higher than those in the healthy cattle.

KEY WORDS: caudal vena cava, heart disease, radiographic parameter.

J. Vet. Med. Sci. 68(9): 995-998, 2006

The vena cava is a highly compliant low-pressure capacity vessel [10]. Its dimensions and dynamics vary with changes in total body water and circulatory blood volume $[13,14,16]$, and may be influenced by the systemic and hepatic circulatory status [3], the compliance of the hepatic parenchyma [27], and changes in the thoracic and abdominal pressure [19].

In human medicine, the inferior vena cava (IVC) corresponds to the caudal vena cava (CVC) of cows, and both the diameter and collapsibility index (CI, called the pulsation index in cattle) of the IVC are important for diagnosis of circulation dysfunction. Measurement of the IVC has been reported during diagnosis of patients with cardiac disease classically associated with right-sided congestive heart failure, including severe or chronic congestive heart failure [9, $12]$, tricuspid incompetence $[20,26]$, and pericardial effusion $[6,20]$. Furthermore, the dilatation of the IVC is also observed in patients with cirrhotic portal hypertension [27]. Measurement of the IVC has been applied to assess right heart function [18], the risk of pregnancy-induced hypertension [22], and to monitor the effect of therapy in patients with congestive heart failure [17].

In small animal clinics, dilatation of the CVC is often listed as an indicator of right-sided congestive heart failure $[8,15]$ and is applied to the diagnosis of dogs with that type of condition, including heartworm disease [2], pericardial disease [8], pulmonic stenosis [23], tricuspid valve regurgitation, and dilated cardiomyopathy [15]. In cattle, with the exception of Braun et al. [3, 4], no one has described alteration of the CVC secondary to poisoning or thrombosis of

\footnotetext{
* Correspondence to: Miyahara, K., Veterinary Medical Teaching Hospital, Obihiro University of Agriculture and Veterinary Medicine, Obihiro, Hokkaido 080-8555, Japan.
}

the CVC, and the dimensions and dynamics of the CVC have not been documented systematically. The aim of this study was to evaluate the radiographic parameters of the CVC in healthy dairy cattle and demonstrate their usefulness in clinical diagnosis.

Prior to the experiment, 81 Holstein cows were subjected to serological and radiological examinations to exclude circulation dysfunction and other diseases. Then, according to the "Standard Growth Values of Holstein Dairy Cattle" [25] cattle were divided into two groups-growing $(\mathrm{n}=43$; aged 9 to 35 months; mean \pm SD: $18.72 \pm 8.16$ ) and adult cows $(n=38$; aged 37 to 95 months; mean \pm SD: $60.87 \pm 15.84)$. The test group, which included 10 cows (aged 26 to 73 months; mean \pm SD: $43.9 \pm 17.34$ ) suspected of heart disease based on clinical symptoms, serological tests, and electrocardiographic records, was carried into our teaching hospital for necropsy. Appearance at necropsy demonstrated 5 cows with vegetative endocarditis, 1 cow with traumatic pericarditis, 2 cows with septal defect, and 2 cows with pericardial effusion.

After each cow was placed on an X-ray vehicle, and restrained in the standing position in the middle of the frame, radiographic examinations were performed using an industrial fluoroscope (MG321; Hitachi Medico, Tokyo) with camera loaded on an X-ray mass screening car for large animals. Radiographic images were taken from the right side of the standing cattle, and Lehmkuhl's method[15] was employed to measure the following: 1) maximum and minimum diameter of the CVC (CVCmax and CVCmin) at the end of expiration and 2) the length of the thoracic vertebrae (VL, usually the 8th thoracic vertebra) and diameter of the aorta (Ao) for comparison as body structures considered not directly affected by alteration of the $\mathrm{CVC}$, but positioned in the same intercostal space as the location of CVC measure- 
ments. From these measurements, the average diameter of the CVC (CVCave) was calculated as (CVCmax + CVC$\min ) / 2$. The pulsation index of the CVC (CVCp.i.) was calculated as (CVCmax-CVCmin)/CVCmax $\times 100 \%$. The CVCave/Ao, CVCave/VL, and Ao/VL ratios were then worked out. Linear regression analyses and independentsample t-tests were performed to analyze all related data using the SPSS analysis software (SPSS 12.0.J for Windows Advanced Models, SPSS Inc., Japan). $p$ values of less then 0.05 were considered statistically significant.

As shown in Table 1, CVCmax was significantly lower in growing cows than in adult cows $(p<0.01)$. There was no difference detected for CVCmin between the 2 groups of cattle. However, the average diameter and pulsation indices of the CVC were significantly lower in the growing cows than in the adult cows $(p<0.01$ both). Age, Ao, and VL were significantly lower in the growing cows than in the adult cows $(p<0.01)$. According to the Holstein Cattle Association of Japan (HCAJ), Holstein cows reach $90 \%$ of their mature body characteristics at approximately 36 months of age [25]. In the present study, adult Holstein cows aged over 36 months are considered to be of mature weight. In regard to the other characteristics, the Ao and VL8 values were related to growth; thus, growing cows had significantly lower Ao and VL8 values than the adult cows. The differences in CVCmax and CVCp.i, between growing and adult cows are not only affected by growth, but also by circulating blood volume. In the lactating period, it is well recognized that the blood volume and cardiac output of cows is increased simultaneous to an increase in the distribution of blood volume flowing into the mammary glands for milk production $[11,21]$. However, in the present study, no significant correlation was detected between the CVC size and monthly daily milk yield. In human medicine, the collapsibility index of the IVC has been correlated with the central venous pressure [24]. The dimensions and collapsibility index of IVC has been demonstrated to be influenced by total body fluid, circulation blood volume, and intrathoracic and intra-abdominal pressure $[1,5,7,14,16]$.

Individual differences between cows prohibit comparison of absolute CVC size. However, the ratio of CVC to other anatomic structures may provide a useful assessment. Therefore, quantitative evaluation was performed to compare the average diameter of the CVC with the diameter of the aorta and length of the 8th thoracic vertebra. As shown in Table 1, the ratio of CVCave to the aorta (CVCave/Ao) was $0.62 \pm 0.09$ (ranged from 0.43 to 0.79 ) in the adult cows and $0.60 \pm 0.11$ (ranged from 0.39 to 0.90 ) in the growing cows. The ratio of CVCave to length of the 8th thoracic vertebra (CVCave/VL) was $0.42 \pm 0.06$ (ranged from 0.25 to 0.52 ) in the adult cows and $0.41 \pm 0.06$ (ranged from 0.25 to 0.59 ) in the growing cows. The t-test showed that the CVCave/Ao and CVCave/VL ratios were consistent in both groups, and their values were $0.61 \pm 0.10$ and $0.41 \pm 0.06$ in all healthy cows, respectively. On the other hand, as shown in Fig. 1A and B, linear regression analyses used to evaluate the correlation of CVCave to Ao and VL for the cattle revealed significant coefficients in both groups examined. The values of $\mathrm{r}^{2}$ for linear regression analyses of Ao and VL were 0.29 and 0.36 for all cattle $(p<0.01)$, respectively.

Although the diameter and pulsation index of the CVC differ significantly between growing and adult cows, the average diameter of the CVC correlates with the size of the Ao and VL in growing and adult cows. The CVCave/Ao and CVCave/VL ratios were fixed values in both the growing and mature cattle. To demonstrate the clinical application of these results as a parametric ruler of the caudal vena cava in healthy cattle, 81 healthy cattle were treated as a control group and 10 cattle with heart disease were used as the test group.

Due to the significant difference in average diameter and pulsation index of the CVC in growing and adult cows, the average diameter of the CVC for the test group was compared with the growing and adult cows of the control group,

Table 1. Mean \pm SD values for age, diameter of aorta, length of 8 th thoracic vertebrae, maximum, minimum, average diameter, and pulsation index of the caudal vena cava, ratios of the caudal vena cava to the aorta and 8th thoracic vertebra, and ratio of the aorta to the 8th thoracic vertebra for the two groups of cows

\begin{tabular}{lcccc}
\hline & Growing $(\mathrm{n}=43)$ & Adult $(\mathrm{n}=38)$ & All $(\mathrm{n}=81)$ & $p^{*}$ \\
\hline Age $(\mathrm{mm})$ & $18.72 \pm 8.16$ & $60.87 \pm 15.84$ & $38.49 \pm 24.47$ & $1.44 \mathrm{E}-20$ \\
Ao $(\mathrm{mm})$ & $36.15 \pm 4.13$ & $40.92 \pm 4.96$ & $38.39 \pm 5.10$ & $1.34 \mathrm{E}-05$ \\
VL $(\mathrm{mm})$ & $52.73 \pm 4.46$ & $60.86 \pm 5.27$ & $56.55 \pm 6.32$ & $1.58 \mathrm{E}-10$ \\
CVCmax $(\mathrm{mm})$ & $25.10 \pm 4.70$ & $30.34 \pm 5.38$ & $27.82 \pm 5.69$ & $1.20 \mathrm{E}-05$ \\
CVCmin $(\mathrm{mm})$ & $17.98 \pm 4.11$ & $19.62 \pm 3.93$ & $18.83 \pm 4.08$ & 0.07 \\
CVCave $(\mathrm{mm})$ & $21.60 \pm 4.25$ & $25.27 \pm 3.99$ & $23.32 \pm 4.41$ & $1.06 \mathrm{E}-04$ \\
CVCp.i. & $28.28 \pm 7.57$ & $34.42 \pm 13.43$ & $31.16 \pm 11.09$ & 0.008 \\
CVCave/Ao & $0.6 \pm 0.10$ & $0.62 \pm 0.09$ & $0.61 \pm 0.10$ & 0.31 \\
CVCave/VL & $0.41 \pm 0.06$ & $0.42 \pm 0.06$ & $0.41 \pm 0.06$ & 0.67 \\
\hline
\end{tabular}

*The value of $p$ is shows the difference in CVCmax, CVCmin, CVCave, CVCp.i., CVCave/Ao, CVCave/VL, and Ao/VL for the growing and adult. Ao = Diameter of aorta. VL = Length of the 8 th thoracic vertebra. CVCmax = Maximum diameter of the caudal vena cava. CVCmin = Minimum diameter of the caudal vena cava. $\mathrm{CVCave}=$ Average diameter of the caudal vena cava. CVCp.i. $=$ Pulsation index of the caudal vena cava. CVCave/Ao $=$ Ratio of the caudal vena cava to the aorta. CVCave/VL = Ratio of the caudal vena cava to the 8 th thoracic vertebra. Ao/VL= Ratio of the aorta to the 8 th thoracic vertebra. 

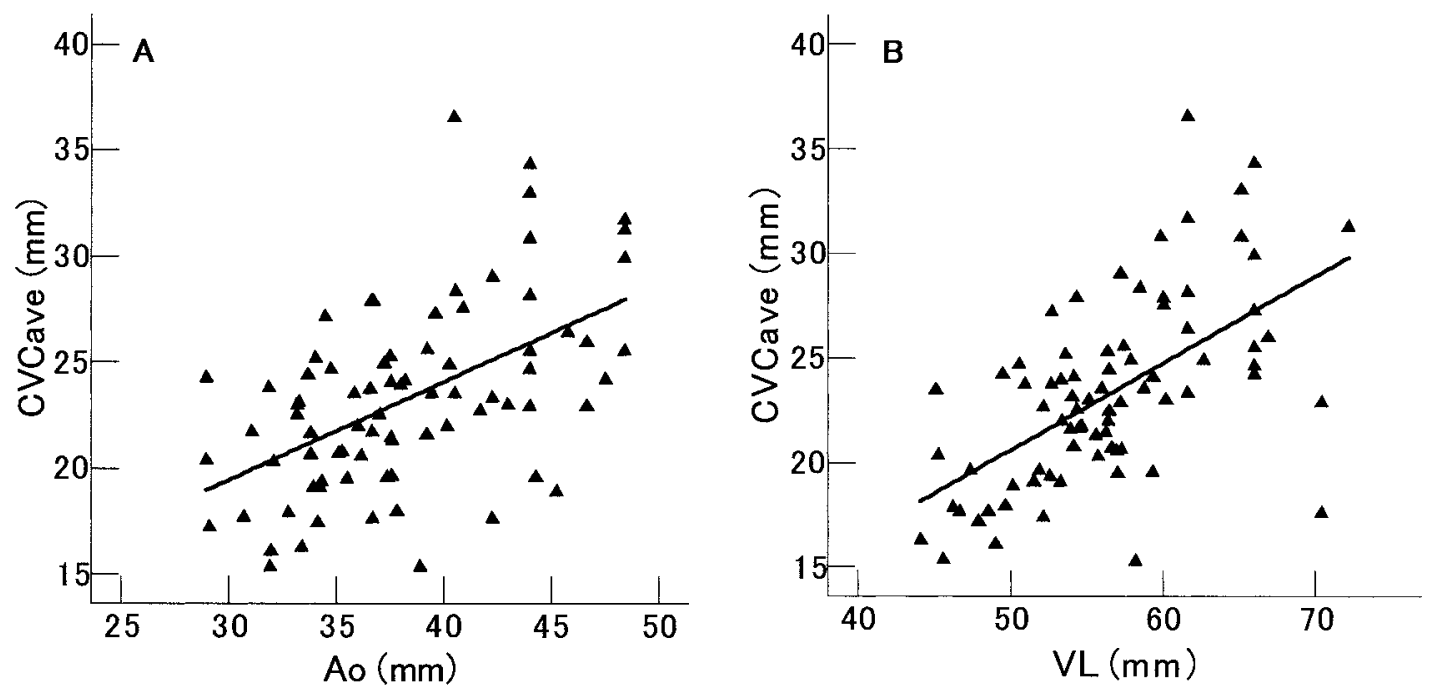

Fig. 1. Correlations between diameters of the caudal vena cava (CVCave) and diameter of the aorta (Ao) or length of the 8th thoracic vertebrae (VL8). A: Graph depicting linear regression analyses between diameters of the caudal vena cava (CVCave) and diameter of the aorta (Ao). CVCave $=5.56+0.46 \times A o, \mathrm{r}^{2}=0.29, \mathrm{n}=81, p<0.01$. B: Graph depicting linear regression analyses between diameters of the caudal vena cava (CVCave) and length of the 8th thoracic vertebrae (VL8), CVCave $=-0.21+0.42 \times \mathrm{VL} 8, \mathrm{r}^{2}=0.36, \mathrm{n}=81, p<0.01$.

A

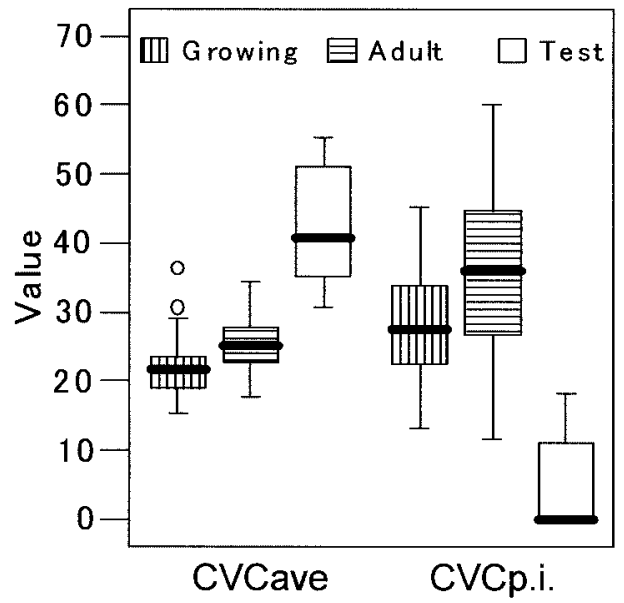

B

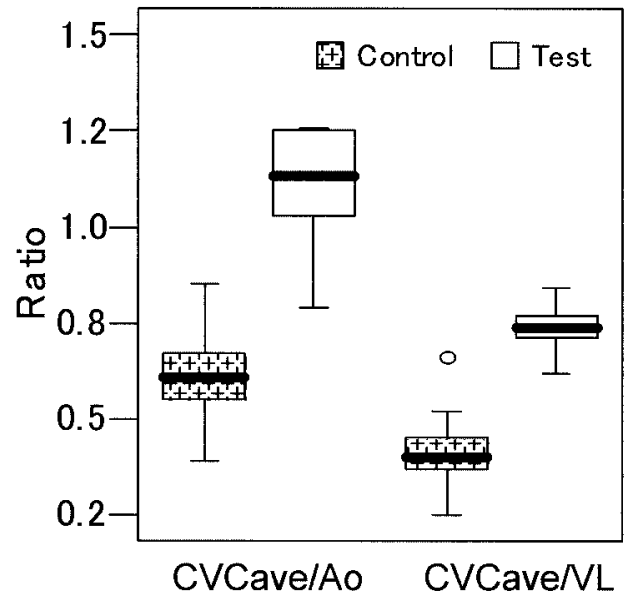

Fig. 2. Comparison of each parameter between the control cows and cows with heart diseases. A: The average diameter (CVCave) and pulsation index of the CVC (CVCp.i.) in growing $(n=43)$, adult $(n=38)$, and cows with heart disease $(\mathrm{n}=10)$. The boxplots display the median, 25th percentile, 75th percentile, and smallest and largest values. Outliers (outside values) are designated with a dot. B: Ratios of CVCave to Ao and VL in the controls $(\mathrm{n}=81)$ and cows with heart disease $(\mathrm{n}=10)$. The boxplots display the median, 25th percentile, 75 th percentile, and smallest and largest values. Outliers (outside values) are designated with a dot.

respectively. As shown in Fig. 2, the average diameter of the CVC in the test group $(42.13 \pm 8.9$, mean $\pm \mathrm{SD})$ was significantly greater than in the growing $(21.54 \pm 4.25)$ and adult cows $(24.98 \pm 3.93)$ (both $p<0.0001)$ of the control group. In the test group, the pulse of the CVC was extremely weak in 3 cows and disappeared in 7 cows, and the pulsation index of the CVC $(4.20 \pm 6.99)$ was signifi- cantly lower than that of growing cows $(27.81 \pm 7.38)$ and adult cows $(34.27 \pm 13)$ (both $\mathrm{p}<0.0001)$ (Fig. $2 \mathrm{~A})$. No difference was detected between the test and control groups in thoracic vertebrae length (test $=57.41 \pm 61.03$, control= $56.70 \pm 41.66)$ or diameter of the aorta (test $=38.17 \pm 48.28$, control $=38.51 \pm 27.45$ ). However, the ratios of CVCave to the aorta and length of the thoracic vertebra (fixed value in 
control groups) were significantly larger in the test cows compared with the control group (CVCave/Ao: test $=1.11 \pm$ 0.15 , control $=0.61 \pm 0.10 ;$ CVCave/VL: test $=0.73 \pm 0.06$, control $=0.41 \pm 0.06$ ) (both $p<0.0001)$ (Fig. 2B). In this study, the test cows had a lower pulsation index and larger caudal vena cava than the control cows as determined by the CVCave/Ao and CVCave/VL ratios. Our results are consistent with previous studies performed in human and small animals $[8,9,12,15,20,26]$, which had similar results.

In conclusion, the pulsation index of the CVC and the ratios of CVCave to the diameter of the aorta and length of the 8 th thoracic vertebra are useful parameters for diagnosis of cattle with circulation dysfunction. However, as an industrial animal, adult dairy cows essentially continuously repeat states of reproductive activity. Throughout their entire lives they are pregnant or lactating, and approximately half of this time is subject to both conditions simultaneously. Therefore, additional studies are needed to examine alteration of intravascular pressure and the dimensions and dynamics of the CVC of cattle during the reproductive cycle, including delivery and different lactating stage.

ACKNOWLEDGEMENTS. The authors would like to acknowledge the Field Center of Animal Science and Agriculture of Obihiro University for supplying the basic information for the healthy cattle. The authors thank Tokachi and Nemuro NOSAI for supplying the cows with heart diseases and Prof. Takane Matsui, Dr. Hidefumi Furuoka, and Dr. Yoshiyasu Kobayashi for participating necropsies.

\section{REFERENCES}

1. Ando, Y., Yanagiba, S. and Asano, Y. 1995. Artificial Organs 19: $1237-1242$.

2. Annette, L., Clarke, A., Rick, A. and James, B. 2005. J. Vet. Cardiol. 7: 33-40.

3. Braun, U. and Linggi, T. 1999. Vet. Rec. 144: 122-126.

4. Braun, U., Fluckiger, M., Feige, K. and Pospischil, A. 2002. Vet. Rec. 150: 209-213.

5. Cheriex, E. C., Leunissen, K. M. L., Janssen, J. H. A., Mooy, J. M. V. and Van, H. J.P. 1989. Nephrol. Dial. Transplant. 4:
563-568.

6. Curvo-Semedo, L., Teixeira, L. and Caseiro-Alves, F. 2005. Eur. J. Radiol. 55: 158-172.

7. Duvekot, J. J., Cheriex, E. C., Tan, D. V., Heidendal, G. A. K. and Peeter, L. L. H. 1994. Cardiovasc. Res. 28: 1269-1272.

8. Gidlewski, J. and Petrie, J. P. 2005. Clin. Tech. Small Anim. Pract. 20: 151-155.

9. Goei, R., Ronnen, H. R., Kessel, A. H. and Kragten, J. A. 1997. Rofo Fortschr Geb Rontgenstr Neuen Bildgeb Verfahr. 166: 36-39.

10. Hasegawa, M. M. 1980. Cardioangiol. 7: 110-118.

11. Hirose, H. 2002. Adv. Anim. Cardiol. 35: 48-51.

12. Hollerbach, S., Schultze, K., Muscholl, M. and Schölmerich, J. 2001. Dtsch. Med. Wochenschr 126: 129-133.

13. Katzarski, K. S., Nisell, J., Randmaa, I., Danielsson, A., Freyschuss, U. and Bergstrom, J, 1997. Am. J. Kidney Dis. 30: 459465.

14. Kusaba, T., Yamaguchi, K. and Oda, H. 1994. Jpn. J. Nephrol. 36: 914-920.

15. Lehmkuhl, L. B., Bonagura, J. D., Biller, D. S. and Hartman, W. M. 1997. Vet. Radiol. Ultra. 38: 94-100.

16. Lyon, M., Blaivas, M. and Brannam, L. 2005. Am. J. Emerg. Med. 23: 45-50.

17. Mandelbaum, A. and Ritz, E. 1996. Nephrol. Dial. Transplant. 11: $24-27$.

18. Mintz, G. B., Kotler, M. N., Parry, W. R., Iakandrain, A. S. and Kane, S. A. 1981. Circulation 64: 1018-1025.

19. Natori, H., Tamaki, S. and Kira, S. 1979. Am. Rev. Respirat. Dis. 120: 421-427.

20. Rein, A. J., Lewis N., Forst, L., Gotsman, M. S. and Lewis, B. S. 1982. Isr. J. Med. Sci. 18: 581-585.

21. Reynolds, M. 1953. Am. J. Physiol. 175: 118-122.

22. Ryo, E., Unno, N., Hagino, D., Kozuma, S., Nagasaka, T. and Taketani, Y. 1999. Intern. J. Gynecol. Obstetr. 65: 143-148.

23. Suter, P. F. and Lord, P. F. 1971. J. Am. Vet. Med. Assoc. 158 : 358-371.

24. Tamaki, S. 1981. Nihon Kyobu Shikkan Gakkai Zasshi. 19: 460-469 (in Japanese).

25. The Holstein Cattle Association of Japan.1995. Standard Grows Value of Holstein Dairy Cattle (in Japanese).

26. Vaturi, M., Shapira, Y., Vaknin,A. H., Oron, A., Matesko, R. and Sagie, A. 2003. J. Heart Valve Dis. 12: 197-201.

27. Wachsberg, R. H., Levine, C. D., Maldjian, P. D. and Simmons, M. Z.1998. Clin. Imaging 22: 48-53. 